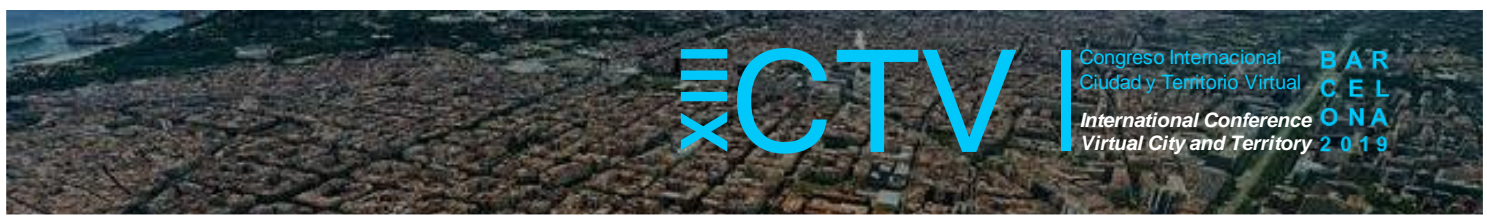

\title{
DESARROLLO DE CAPACIDADES ADAPTATIVAS AL CAMBIO CLIMÁTICO EN COMUNIDADES URBANAS Y RURALES DEL SUR DE JALISCO, MÉXICO
}

Michel Parra, J. Guadalupe ${ }^{1 *}$;Montañez Valdez, Oziel Dante2; Martínez Ibarra, José Alejandro ${ }^{3}$; Orendain Verduzco, Tomás Eduardo4; y Cerón Bracamontes, Miriam ${ }^{5}$

Remisión inicial: 2019-05-31; Remisión definitiva: 2019-10-17; Publicación: 2019-12-21

Citación: Michel Parra, J. G. et al. (2019). Desarrollo de capacidades adaptativas al cambio climático en comunidades urbanas y rurales del sur de Jalisco, México. En XIII CTV 2019 Proceedings: XIII International Conference on Virtual City and Territory: "Challenges and paradigms of the contemporary city": UPC, Barcelona, October 2-4, 2019. Barcelona: CPSV, 2019, p. 8495. E-ISSN 2604-6512.DOI http://dx.doi.org/10.5821/ctv.8495

\section{Resumen}

El presente trabajo tiene un enfoque sistémico de la zona de estudio que está comprendida por los medios urbanos y rurales de los municipios de Zapotitlán de Vadillo, Tuxcacuesco, Zapotlán el Grande, Tuxpan y Gómez Farías, Jalisco, haciendo manifiesto los aspectos ecológicos, sociales, económicos políticos y culturales que se pretenden modificarse mediante la Educación Ambiental formal e informal y toma de conciencia de las vulnerabilidades, riesgos, amenazas que aquejan a estos municipios en diferente grado, así como la repercusión que está teniendo por el cambio climático y el calentamiento global. Es por ello, que las acciones que se llevaron a cabo durante el desarrollo de este proyecto, tratan de socializar, concientizar y educar a los habitantes de esta región para mitigar el impacto negativo y así mejorar su calidad de vida.

Al desarrollar capacidades adaptativas al cambio climático en comunidades urbanas y rurales a través de la Educación Ambiental en cinco municipios de la región Sur de Jalisco.

La zona de estudio se ubica en el Sur del estado de Jalisco, México. En tres cuencas hidrológicas diferentes (Cuenca Armería, Cuenca Lerma-Chapala Santiago, y Coahuayana. Asimismo, en tres regiones, Sierra de Amula, Región Sur y Sureste, las cuales presentan características socio-ambientales distintas con microclimas particulares. Sin embargo, tienen en común aspectos ambientales, sociales, económicos, políticos y culturales, y sobre todo un grado significativo de vulnerabilidad a los diferentes tipos de pobreza según la clasificación de vulnerabilidad al cambio climático en los municipios de México (Consejo Nacional de Evaluación de la Política de Desarrollo Social, 2018). Las interacciones de uno afectan a los demás municipios, por ejemplo, la escasez de agua en una región afectará de manera directa a tres o cuatro municipios aledaños.

Las principales líneas de acción para el desarrollo del proyecto fueron: Educación Ambiental para la sustentabilidad, Capacidades adaptativas para la contribución al cambio climático, Contribuir a la resiliencia de los ecosistemas de la región en estudio, Fortalecimiento en gestión ambiental y los Sistemas productivos básicos con buenas prácticas.

Para el desarrollo de los procesos de aprendizaje social y acción colectiva que orientaron la construcción de capacidades adaptativas integrales fueron a través de: El Aprendizaje Basado en Problemas (ABP), aprendizaje lúdico, desarrollo de vivencias, participación proactiva, talleres, congresos, conferencias, paneles, mesas de trabajo y eventos culturales masivos, Feria del agua, Feria de la tilapia, calendario ambiental: destacando la celebración del día mundial de los humedales, día mundial del agua, de la tierra y medio ambiente, encuentro regional, nacional e internacional de las comunidades indígenas.

Para lo cual, se utilizaron materiales educativos y comunicacionales en el desarrollo del proceso que fueron aulas al aire libre, auditorios, guía de trabajo, visitas guiadas, observación directa, comunicación oral, mímica y escrita,

\footnotetext{
1 Centro de Investigación Lago de Zapotlán y Cuencas, https://orcid.org/0000-0002-7017-8360; 2 Centro de Investigación Lago de Zapotlán y Cuencas, https://orcid.org/0000-0001-9539-6623; ${ }^{3}$ Centro de Investigación Lago de Zapotlán y Cuencas, https://orcid.org/0000-0002-6600-654X; ${ }^{4}$ Centro de Investigación Lago de Zapotlán y Cuencas, https://orcid.org/0000-0002-6914-1288 y ${ }^{5}$ Centro de Investigación Lago de Zapotlán y Cuencas, https://orcid.org/0000-0003-3151-236X. * Correo de contacto: michelp@cusur.udg.mx
} 


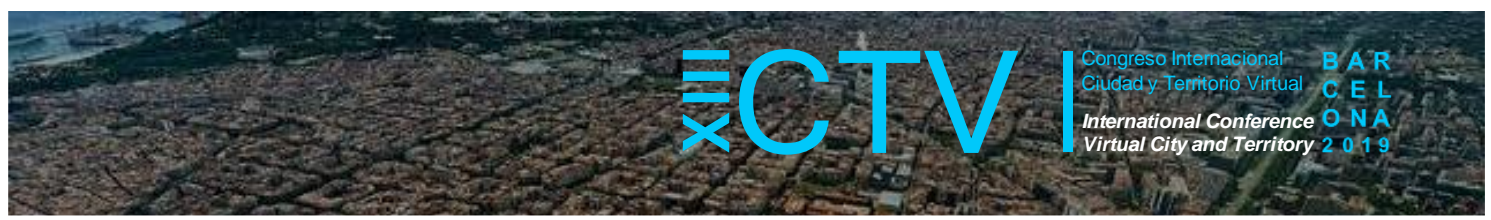

presentación de videos educativos, presentación de power point, materiales electrónicos y técnicas didácticas preinstrucionales, coinstrucional y postinstrucional, carteles y domo didáctico interactivo.

No obstante, la importancia de este trabajo radica en el reconocimiento de la vulnerabilidad de los municipios antes mencionados y su impacto en el corredor socio-ambiental de estos. Al realizar este trabajo de georreferenciación se buscaron los puntos críticos vulnerables que presentan los grupos sociales para llegar a ser más sensibles a los efectos negativos del cambio climático.

En conclusión, existe cierto grado de viabilidad que se puede contribuir a la resiliencia de la región para contribuir a la remediación del cambio climático, ya que es un problema global en el cual se puede contribuir con acciones locales de buenas prácticas en el uso y aprovechamiento racional de los bienes y servicios ecosistémicos de la región; mediante el desarrollo de capacidades adaptabilidad, mediante la educación ambiental formal e informal, lo cual fortalece y fomenta los valores de la sustentabilidad.

En este trabajo participaron 1,978 personas, los cuales son estudiantes de educación básica y de educación media superior, así como productores agropecuarios, forestales y turísticos de los cinco municipios antes mencionados y 50 miembros de la comunidad indígena náhuatl: estudiantes de la telesecundaria del ejido de Zenzontla, del Municipio de Tuxcacuesco, Escuela preparatoria de Tuxcacuesco, Zapotitlán de Vadillo, Ciudad Guzmán, Tuxpan y escuela secundaria de San Sebastián del Sur, Colegio de bachilleres del Estado de Jalisco (COBAEJ), Centro de Bachillerato Tecnológico Industrial y de Servicios (CBTis) en edades de 13 y 18 años.

\section{Abstract}

This paper is a systemic approach to the study area that is covered by urban and rural areas of the municipalities of Zapotitlan de Vadillo, Tuxcacuesco, Zapotlan el Grande, Tuxpan and Gomez Farias, Jalisco, Mexico. Making manifest the ecological, social, economic, political and cultural aspects that to be modified are intended through formal and informal Environmental Education and raising awareness of the vulnerabilities, risks, threats that affect these municipalities to different degrees, as well as the impact they are taking into the climate change and global warming. Through developing adaptive capacities, the many climate changes in urban and rural communities through Environmental Education in five municipalities of the Southern Jalisco region.

The study area is located in the south of the state of Jalisco, Mexico. In three different hydrological basins (Armería Basin, Lerma-Chapala Santiago Basin, and Coahuayana. In addition, in three regions, Sierra de Amula, South and Southeast Region, which have different socio-environmental characteristics with particular microclimates. However, they have common environmental, social, economic, political and cultural aspects, and above all a significant degree of vulnerability to different types of poverty according to the classification of vulnerability to climate change in the municipalities of Mexico (National Institute of Ecology and Climate Change, 2013). The interactions of one affect the other municipalities, for example, water scarcity in a region will directly affect three or four neighboring municipalities.

The main lines of action for the development of the project was: Environmental education for sustainability, adaptive capacities for contribution to climate change, contribute to the resilience of ecosystems in the region under study, strengthening environmental management and basic production systems good practices.

For the development of the processes of social learning and collective action that guided the construction of integral adaptive capacities were through: Problem-Based Learning (PBL), playful learning, development of experiences, proactive participation, workshops, congresses, conferences, panels, work tables and mass cultural events, Water Fair, Tilapia Fair, environmental calendar: highlighting the celebration of World Wetlands Day, World Water, Earth and Environment Day, regional, national and international meeting of indigenous communities.

For which, educational and communication materials were used in the development process were outdoor classrooms, auditoriums, working guide, guided visits, direct observation, oral, mimicry and written communication, presentation of educational videos, power point presentation, electronic materials and pre-instructional, coininstructional and post-instructional teaching techniques, posters and interactive dome.

However, the importance of this work is in recognition of the vulnerability of the aforementioned municipalities and their impact on the socio-environmental corridor of these. When performing this work georeferencing vulnerable critical points that have social groups to become more sensitive to the negative effects of climate change sought were.

In conclusion, there is some degree of viability can contribute to the resilience of the region to contribute to the remediation of climate change because, it is a global problem which can contribute to local actions of good practice in 


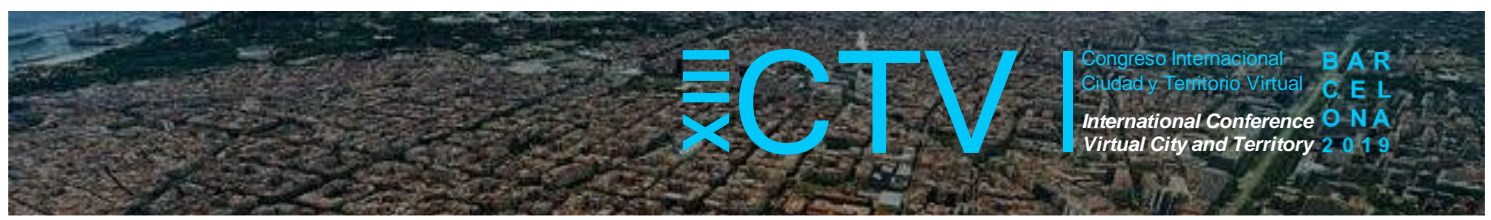

the use and exploitations rational use of ecosystem goods and services in the region; by developing adaptability skills through formal and informal environmental education, which strengthens and promotes the values of sustainability.

In this work they involved 1,978 people, who are students of basic education and higher secondary education, as well as the five municipalities mentioned above agricultural, forestry and tourism producers and 50 members of the Nahua indigenous community: students telesecundaria the Zenzontla, from the Municipality of Tuxcacuesco, Tuxcacuesco High School, Zapotitlan de Vadillo, Ciudad Guzmán, Tuxpan and San Sebastian del Sur High School, Jalisco State Baccalaureate College (COBAEJ), Industrial and Baccalaureate Center for Technology and Services (CBTis) between ages 13 and 18. Which showed high availability to continue contributing to formal and informal environmental education with their family, friends and social circles in their environment.

Palabras Clave: Capacidades adaptativas; cambio climático; educación ambiental; vulnerabilidad urbana y rural

Key words: Adaptive capabilities; Climate change; Environmental education; Urban and rural vulnerability

\section{Introducción}

El presente trabajo tiene un enfoque sistémico de la zona de estudio que está comprendida por los medios urbanos y rurales de los municipios de Zapotitlán de Vadillo, Tuxcacuesco, Zapotlán el Grande, Tuxpan y Gómez Farías, Jalisco, haciendo manifiesto los aspectos ecológicos, sociales, económicos políticos y culturales que se pretenden modificarse mediante la Educación Ambiental formal e informal y toma de conciencia de las vulnerabilidad, riesgos, amenazas que aquejan a estos municipios en diferente grado, así como la repercusión que está teniendo por el cambio climático y el calentamiento global. Es por ello que las acciones que se llevarán a cabo durante el desarrollo del proyecto, tratan de socializar, concientizar y educar a los habitantes de esta región para mitigar el impacto negativo y así mejorar su calidad de vida.

El presente trabajo se realizó en la Región Hidrológica número 16 Armería-Coahuayana, la conforman las Subregiones Hidrológicas Río Armería y Río Coahuayana, está ubicada en una zona fisiográfica compleja entre las regiones comprendidas por el eje Neovolcánico, la Sierra Madre del Sur y la Sierra Madre Occidental. Cuenta con una extensión de 17,626.6 kilómetros cuadrados, una longitud total de 240 kilómetros y un volumen anual de escurrimiento de 2,076 millones de metros cúbicos anuales. Así como en el número 12 Cuenca Hidrológica LermaChapala- Sayula - Zapotlán (Comisión Nacional del Agua, 2017).

La zona de estudio se ubica en el Sur del estado de Jalisco, México. En tres cuencas hidrológicas diferentes (Cuenca Armería, Cuenca Lerma-Chapala Santiago, y Coahuayana. Asimismo, en tres regiones, Sierra de Amula, Región Sur y Sureste, las cuales presentan características socio-ambientales distintas con microclimas particulares. Sin embargo, tienen en común aspectos ambientales, sociales, económicos, políticos y culturales, y sobre todo un grado significativo de vulnerabilidad según la clasificación de vulnerabilidad al cambio climático en los municipios de México (Instituto Nacional de Ecología y Cambio Climático, 2013). Las interacciones de uno afectan a los demás municipios, por ejemplo, la escasez de agua en una región afectará de manera directa a tres o cuatro municipios aledaños.

Para lo cual, se utilizaron materiales educativos y comunicacionales en el desarrollo del proceso que fueron aulas al aire libre, auditorios, guía de trabajo, visitas guiadas, observación directa, comunicación oral, mímica y escrita, presentación de videos educativos, presentación de power point, materiales electrónicos y técnicas didácticas preinstrucionales, coinstrucional y posinstrucional, carteles y domo didáctico interactivo. 


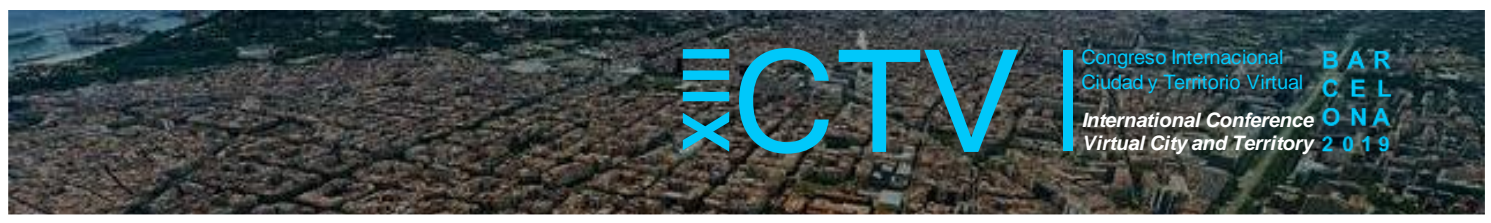

No obstante, la importancia de este trabajo radica en el reconocimiento de la vulnerabilidad de los municipios antes mencionados y su impacto en el corredor socio-ambiental de estos. Al realizar este trabajo de georreferenciación se buscaron los puntos críticos vulnerables que presentan los grupos sociales para llegar a ser más sensibles a los efectos negativos del cambio climático.

Ya que el artículo 27 constitucional señala, que todos los mexicanos tenemos el compromiso de contribuir a la protección, conservación y manejo sustentable de los humedales para que puedan cumplir con sus bienes y servicios ecosistémicos (Constitución Política de los Estados Unidos Mexicanos, 2017).

\section{Objetivos}

\subsection{Objetivo general}

Desarrollar capacidades adaptativas al cambio climático en comunidades urbanas y rurales a través de la Educación Ambiental en cinco municipios de la región Sur de Jalisco.

\subsection{Objetivos específicos}

- Realizar monitoreo físicos, químicos y biológicos en las cuencas hidrológicas de los cinco municipios que conforman el área de estudio.

- Fortalecer la Educación Ambiental formal e informal, mediante la producción, alimentación y nutrición mediante la Unidad de producción, educación e investigación de Zenzontla, municipio de Tuxcacuesco.

- Identificar el área vulnerable en los municipios de la zona de estudio.

- Desarrollar actividades lúdicas de Educación Ambiental para crear sentido de pertenencia y gobernabilidad de los grupos involucrados y comprometidos con la protección de los ecosistemas.

- Realizar recorridos temáticos en los cinco municipios del área de estudio

- Implementar actividades de Educación Ambiental para la protección, conservación y manejo de los ecosistemas de las cuencas.

- Contribuir con actividades productivas sustentables en el uso y aprovechamiento del agua, suelo, aire y biodiversidad para mejorar la alimentación, educación y cultura.

\section{Metodología}

El presente trabajo se realizó en la Región Hidrológica número 16 Armería-Coahuayana, la conforman las Subregiones Hidrológicas Río Armería y Río Coahuayana, está ubicada en una zona fisiográfica compleja entre las regiones comprendidas por el eje Neovolcánico, la Sierra Madre del Sur y la Sierra Madre Occidental. Cuenta con una extensión de 17,626.6 kilómetros cuadrados, una longitud total de 240 kilómetros y un volumen anual de escurrimiento de 2,076 millones de metros cúbicos anuales. Así como en el número 12 Cuenca Hidrológica LermaChapala- Sayula - Zapotlán (Constitución Política de los Estados Unidos Mexicanos, 2017).

Los cinco municipios donde se realizó el estudio son: Zapotlán el Grande y Tuxpan, Zapotitlán de Vadillo, Tuxcacuesco y Gómez Farías. Localizados en la región sur del estado de Jalisco, México. 


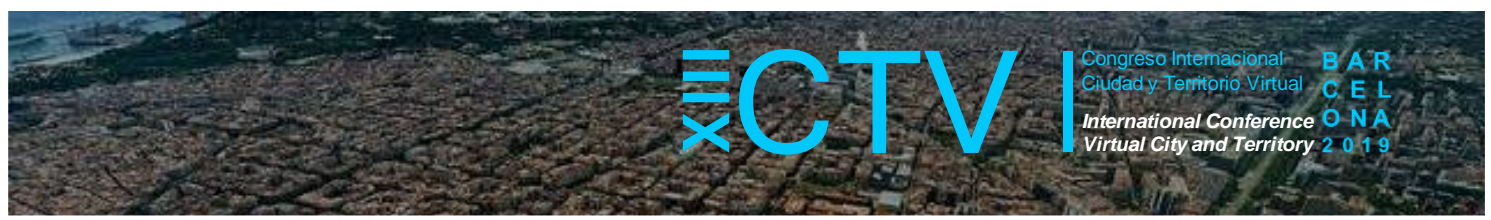

El municipio de Tuxcacuesco se localiza en la región de Amula del estado, Jalisco en la Cuenca Hidrológica Armería entre las coordenadas 190 33' 50" a 19 48' 55" de latitud norte y los $103^{\circ} 52^{\prime} 45^{\prime \prime}$ a los $104^{\circ} 08^{\prime} 40^{\prime \prime}$ de longitud oeste, a una altura de 750 metros sobre el nivel del mar. esta localidad tiene una extensión territorial de $428.82 \mathrm{~km}^{2}$.

La localidad donde se incidió es en la cabecera municipal denominada Tuxcacuesco y el ejido de Zenzontla, se incidió con 200 estudiantes participantes y 30 productores agropecuarios, forestales y turísticos. Zapotitlán de Vadillo se localiza en el centro sur del estado de Jalisco; sus coordenadas son $19^{\circ} 25^{\prime} 00^{\prime \prime}$ y $19^{\circ} 37^{\prime} 50^{\prime \prime}$ de latitud norte y $103^{\circ} 36^{\prime} 50^{\prime \prime}$ y $103^{\circ} 53^{\prime} 55^{\prime \prime}$ de longitud oeste, con una altitud promedio de 1,500 metros sobre el nivel del mar. Su extensión territorial es de $480.74 \mathrm{~km}^{2}$. La cabecera municipal es Zapotitlán, donde se incidió con 169 participantes estudiantes y 30 productores agropecuarios, forestales y turísticos es la localidad donde se realizó dicho trabajo.

Tuxcacuesco se localiza en la región de Amula del estado de Jalisco en la Cuenca Hidrológica Armería, entre las coordenadas $19033^{\prime} 50^{\prime \prime}$ a $19^{\circ} 48^{\prime} 55^{\prime \prime}$ de latitud norte y los $103^{\circ} 52^{\prime} 45^{\prime \prime}$ a los $104^{\circ} 08^{\prime} 40^{\prime \prime}$ de longitud oeste, a una altura de 750 metros sobre el nivel del mar. Este municipio tiene una extensión territorial de $428.82 \mathrm{~km}^{2}$. La localidad donde se incidió es en la cabecera municipal denominada Tuxcacuesco y el ejido de Zenzontla con 200 participantes estudiantes y 30 productores agropecuarios, forestales y turísticos. Zapotlán el Grande se ubica entre las coordenadas $19^{\circ} 34^{\prime} 12^{\prime \prime}$ y $19^{\circ} 46^{\prime} 00^{\prime \prime}$ de latitud norte y $103^{\circ} 23^{\prime} 00^{\prime \prime}$ a los $103^{\circ} 38^{\prime} 00^{\prime \prime}$ de longitud oeste a una altura promedio de 1,580 metros sobre el nivel del mar. Tiene una superficie de $295.29 \mathrm{~km}^{2}$. Colinda con un total de seis municipios: al norte con Gómez Farías; al este con Tamazula de Gordiano; al sureste con Zapotiltic; al sur con Tuxpan; al suroeste con Zapotitlán de Vadillo y al oeste con San Gabriel. Dicho municipio cuenta con un ordenamiento ecológico y territorial (SEMARNAT - SEMADES, 2014). La localidad donde se incidió es en la cabecera municipal denominada Ciudad Guzmán con 1,030 estudiantes participantes y 30 productores agropecuarios, forestales y turísticos.

Gómez Farías se localiza en las coordenadas de los $19^{\circ} 47^{\prime} 15^{\prime \prime}$ a $19^{\circ} 57^{\prime} 30^{\prime \prime}$ de latitud norte y de los $103^{\circ} 09^{\prime} 10^{\prime \prime}$ a los $103^{\circ} 39^{\prime} 20^{\prime \prime}$ de longitud oeste y a una altura de 1,678 metros sobre el nivel del mar. Su extensión territorial es de $343.89 \mathrm{~km}^{2}$. La localidad donde se incidió es en la cabecera municipal denominada: San Sebastián del Sur con 200 participantes estudiantes y 30 productores agropecuarios, forestales y turísticos. Tuxpan se localiza al sureste de Jalisco y está dentro de la Cuenca Hidrológica Coahuayana en las coordenadas extremas $19^{\circ} 14^{\prime} 45^{\prime \prime}$ a los 19 $37^{\prime} 30^{\prime \prime}$ de latitud norte y de los $103^{\circ} 19^{\prime} 00^{\prime \prime}$ a los $103^{\circ} 36^{\prime} 15^{\prime \prime}$ de longitud oeste, a una altura de 1,737 metros sobre el nivel del mar. Su extensión territorial es de $550.23 \mathrm{~km} 2$. La localidad donde se incidió es en la cabecera municipal denominada Tuxpan y Rancho Nuevo, con 200 estudiantes participantes, 50 personas de la comunidad indígena y 30 productores agropecuarios, forestales y turísticos.

Las principales líneas de acción para el desarrollo del proyecto fueron:

- Educación Ambiental para la sustentabilidad

- Capacidades adaptativas para la contribución al cambio climático.

- Contribuir a la resiliencia de los ecosistemas de la región en estudio.

- Fortalecimiento en gestión ambiental.

- Sistemas productivos básicos con buenas prácticas

Para el desarrollo de los procesos de aprendizaje social y acción colectiva que orientaron la construcción de capacidades adaptativas integrales fueron a través de: Los Aprendizaje Basado en Problemas (ABP), aprendizaje lúdico, desarrollo de vivencias, participación proactiva, talleres, congresos, conferencias, paneles, mesas de trabajo y eventos culturales masivos, Feria del agua, Feria de la tilapia, calendario ambiental: destacando la celebración del día mundial de los humedales, día mundial del agua, de la tierra y medio ambiente, encuentro regional, nacional e internacional de las comunidades indígenas. 


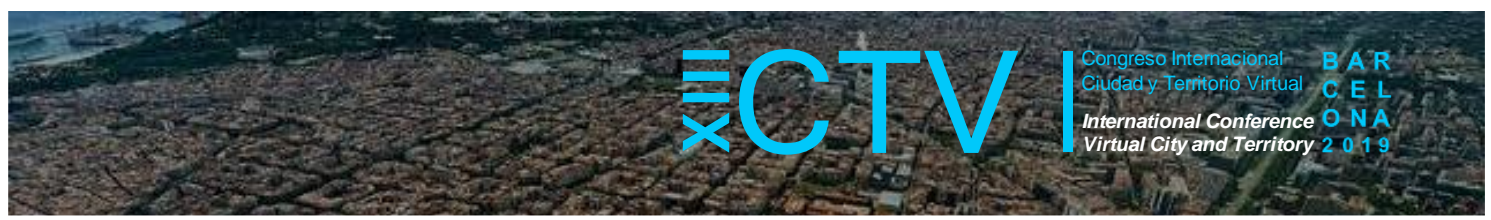

Para lo cual, se utilizaron materiales educativos y comunicacionales en el desarrollo del proceso que fueron aulas al aire libre, auditorios, guía de trabajo, visitas guiadas, observación directa, comunicación oral, mímica y escrita, presentación de videos educativos, presentación de power point, materiales electrónicos y técnicas didácticas preinstrucionales, coinstrucional y posinstrucional, carteles y domo didáctico interactivo.

\section{Resultados y discusiones}

El cambio climático y el calentamiento global están generando una alta vulnerabilidad en la perdida de los recursos naturales, humanos, agrícolas, pecuarias y forestales, así como la generación de fuentes de empleos, problemas de salud y desnutrición, aunado ello se suscitan los incrementos en incendios forestales, con alta perdida de la biodiversidad faunística y florística, los eventos volcán de fuego activo genera emisiones periódicas de cenizas y vapor de agua, movimientos tectónicos, agrietamientos del suelo, derrumbes y deslaves lo cual representan problemáticas significativas para los pobladores de los municipios de la región sur y sureste de Jalisco, los municipios más marginados son los más representativos, donde existe mayor pobreza, falta de educación y marginación social.

Los equipos multidisciplinarios e interinstitucionales que participaran son: Centro Universitario del Sur, Secretaria de Educación Pública, Escuelas Preparatorias y módulos de la Universidad de Guadalajara, Ayuntamientos municipales 2015-2018, Asociación de productores agrícolas, pecuarios, forestales y turísticos de los cinco municipios participantes y comunidad indígena náhuatl de Tuxpan, Jalisco. Organismo Público Descentralizado, Sistema de Agua Potable de Zapotlán (SAPAZA).

El municipio de Zapotitlán de Vadillo, es el más vulnerable conforme a la clasificación de vulnerabilidad al cambio climático en los municipios de México (Instituto Nacional de Ecología y Cambio Climático, 2013).

En Zapotitlán de Vadillo el 77.0 por ciento de la población se encuentra en situación de pobreza, es decir, 7,190 personas comparten esta situación en el municipio, así mismo el 16.0 por ciento ( 1,493 personas) de la población es vulnerable por carencias sociales; el 4.1 por ciento es vulnerable por ingresos y un 2.9 por ciento es no pobre y no vulnerable. Es importante agregar que el 20.0 por ciento, de Zapotitlán de Vadillo presentó pobreza extrema, es decir, 1,867 personas, y un 57.0 por ciento en pobreza moderada (5,323 personas). De los indicadores de carencias sociales, destaca el acceso a la seguridad social es la más alta con un 81.7 por ciento, que en términos relativos se trata de 7,629 habitantes. Los que menos porcentajes acumulan son calidad y espacios de la vivienda y acceso a la alimentación con el 22.9 y 30.3 por ciento respectivamente.

Geográficamente el municipio presenta una topografía de tipo irregular, ya que se encuentra en la parte poniente de la falda del Volcán de fuego y el Nevado de Colima. En la parte central tiene unas altitudes que van de los 300 a los 1,500 metros, mismas que se elevan en el extremo noroeste y sureste, en donde varían entre 2,400 y 3,200 metros, siguiendo hasta las partes más altas del volcán, donde alcanzan hasta los 3,600 metros. El suelo de Zapotitlán es muy accidentado (40\% del territorio), está cruzado por muchas barrancas, pero también tiene altas serranías. Hay pocas zonas planas o llanuras (22\% del territorio), pero hay muchas lomas (zonas semiplanas que representan el $38 \%$ del territorio).

La mayor parte del suelo tiene un uso agropecuario. Debido a la variedad que presenta la superficie terrestre, conformada por barrancas, arroyos, laderas y lomas, se siembra diferentes cultivos maíz, frijol, calabaza, chile y maguey mezcalero.

La riqueza natural con que cuenta el municipio está representada por 18,900 hectáreas de bosque, donde predominan especies de pino, cedro, encino y roble principalmente y 


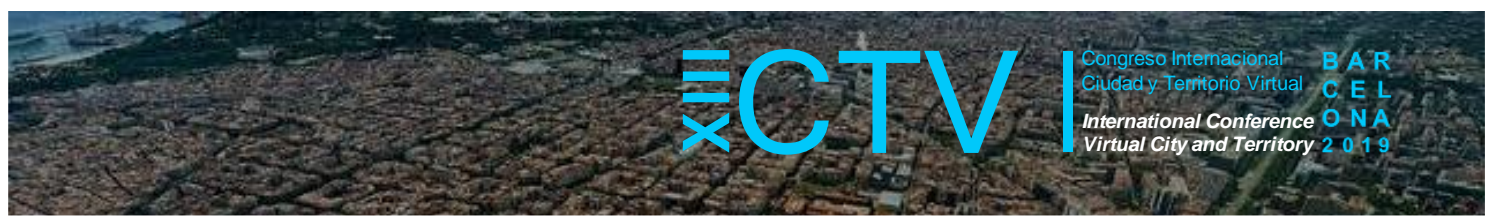

corresponden a parte del Parque Nacional Nevado de Colima, Área Natural Protegida (ANP, federal) y bosque mesofiló o bosque de niebla (ANP, Estatal). Se cría ganado bovino de carne y leche, porcino, caprino, abejas, aves de carne y postura. Se explota principalmente los bosques de coníferas, como fresno, pino, oyamel y encino. Su carencia más importante es el recurso de agua, ya que las condiciones de sus suelos no les permiten buena retención y en gran parte depende de las épocas de lluvia de junio a octubre y de las escorrentías del río Armería.

En el municipio de Tuxcacuesco el 41.5 por ciento de la población se encuentra en situación de pobreza, es decir, 1,872 personas comparten esta situación en el municipio, así mismo el 51.3 por ciento (2,314 personas), de la población es vulnerable por carencias sociales; el 1.3 por ciento es vulnerable por ingresos y 6.0 por ciento es no pobre y no vulnerable. Es importante agregar que el 6.8 por ciento de Tuxcacuesco presentó pobreza extrema, es decir 309 personas, y un 34.6 por ciento en pobreza moderada (1,564 personas). De los indicadores de carencias sociales, destaca que el acceso a la seguridad social es la más alta con un 74.2 por ciento, que en términos absolutos se trata de 3,350 habitantes. Los que menos porcentajes acumulan son calidad y espacios de la vivienda, y acceso a la alimentación con el 13.8 por ciento y 22.6 por ciento respectivamente.

Para el aspecto ambiental, el municipio presenta una topografía muy accidentada ( $70 \%$ del territorio), en la que las máximas alturas se localizan en la parte sur del territorio. De esta parte, hacia el norte, las latitudes van disminuyendo, predominando en la parte central, alturas medias (14\% del territorio) y en la parte norte las más bajas (que cubren el $16 \%$ del municipio). La riqueza natural está representada por 8,015 hectáreas de bosque, donde predominan especies de pino, encino y huizache.

La mayor parte del suelo tiene un uso agrícola. La tenencia de la tierra en su mayoría corresponde a la propiedad ejidal. En los cultivos locales destaca la sandía, maíz, sorgo, melón, jitomate, chile verde y cebolla, recientemente uva, actualmente destacan la agricultura bajo techo para jitomate, desarrollada por empresas nacionales y trasnacionales. Se cría ganado bovino de carne y leche, porcino, caprino, abejas, aves de carne y postura y un poco de pesca.

De los principales problemas que presenta este municipio es la inseguridad, la delincuencia y la pobreza. Lo cual afecta la situación alimentaria encontrándose altos grados de desnutrición y problemas de aprendizaje en los niños.

Zapotlán el Grande es un municipio de muy bajo nivel de vulnerabilidad, ya que se sustenta con una ciudad universitaria donde se concentra la educación media y superior, lo cual concentra los estudiantes de 28 municipios de la región sur y sureste del estado de Jalisco. Sin embargo, su importancia dentro de la zona de estudio radica en la interacción ambiental, educativa, social, económica entre otras, que representa una zona geoeconómica y política de la región para los demás municipios.

En Zapotlán el Grande el 21.7 por ciento de la población se encuentra en situación de pobreza, es decir, 24,037 personas comparten esta situación en el municipio, así mismo el 40.1 por ciento $(44,322$ personas) de la población es vulnerable por carencias sociales; el 5.7 por ciento es vulnerable por ingresos y el 32.5 por ciento es no pobre y no es vulnerable. Es importante agregar que tan solo el 1.7 por ciento de Zapotlán el Grande, presentó pobreza extrema, es decir, 1, 605 personas, y un 20.3 por ciento en pobreza moderada $(22,432$ personas). De los indicadores de carencias sociales, destaca que el acceso a la seguridad social es la más alta con un 43.4 por ciento, que en términos absolutos se trata de 48,003 habitantes. Los que menos porcentajes acumulan son la calidad y espacios, asimismo, el acceso a los servicios básicos de la vivienda, con el 6.4 por ciento y 3.2 por ciento respectivamente. Esto se debe a un alto grado de migración de personas de los estados de 


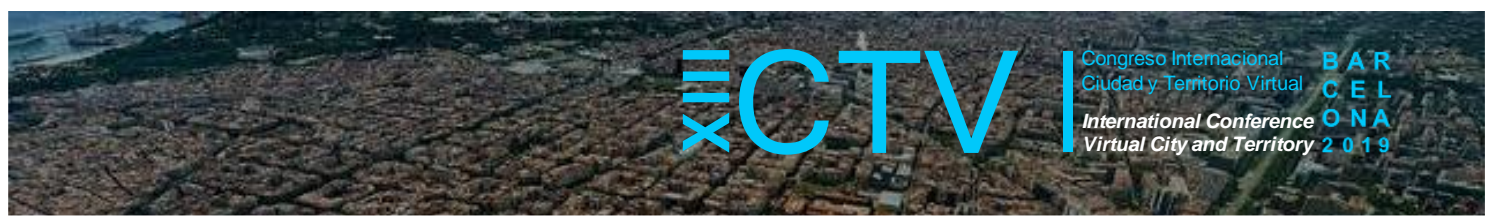

Guerrero, Oaxaca, Chiapas, los cuales buscan trabajo en las empresas de aguacate y berries, las cuales se han acrecentado en los últimos quince años.

Para el municipio el cambio de uso de suelo de bosque nativo a cultivos de aguacate y berries, ha tenido un impacto negativo en la perdida de los ecosistemas y la biodiversidad, así como la carencia de agua con un déficit de 27 millones de metros cúbicos, por consiguiente, si sigue esta dinámica la cuenca tendrá un impacto ambiental, social, político y económico en los próximos diez años (Michel et al, 2018).

Su riqueza natural está representada por 11,200 hectáreas de bosque donde predominan especies de pino, encino y oyamel, principalmente. Sus recursos minerales son yacimientos de arcilla, caliza y yeso. Además, el municipio tiene ramificaciones de la Sierra Volcánica transversal con una altitud de 2,700 metros, dentro de los límites municipales se encuentra parte del Nevado de Colima, donde alcanza una altura de 4,200 metros; el Parque Nacional Nevado de Colima, Área Natural Protegida (ANP, federal) y parte del bosque mesofiló o bosque de niebla (ANP, Estatal) y parte de la "laguna de Zapotlán, sitio Ramsar No 1466, humedal de importancia internacional y corredor migratorio de Aves de Norteamérica y Centroamérica (Ley General del Equilibrio Ecológico y Protección al Ambiente, 2016). Los principales cultivos son: aguacate con 4,000 hectáreas y berries 2,200 hectáreas, con sistema techado; así como maíz, sorgo, frijol en poca cantidad, hortalizas, caña de azúcar. Se tiene ganadería bovina de leche y carne y existen industria de la leche y sus derivados (queso, mantequilla y otros); predomina el ganado bovino, porcino, caprino y un poco lanar.

De las amenazas importantes son el cambio de uso de suelo, el aumento poblacional significativo por migración de jornaleros, la alta demanda de servicios de salud y la presencia de enfermedades de importancia en salud pública como: tuberculosis y brucelosis bovina.

Gómez Farías el 60.0 por ciento de la población se encuentra en situación de pobreza, es decir, 6,797 personas comparten esta situación en el municipio, así mismo el 22.3 por ciento (2,523 personas) de la población es vulnerable por carencias sociales; el 9.7 por ciento es vulnerable por ingresos y 7.9 por ciento es no pobre y no vulnerable. Es importante agregar que el 9.5 por ciento de Gómez Farías presentó pobreza extrema, es decir 1,077 personas, y un 50.5 por ciento en pobreza moderada (5,720 personas). De los indicadores de carencias sociales, destaca que el acceso a la seguridad social es la más alta con un 67.5 por ciento, que en términos absolutos se trata de 7,636 habitantes. Los que menos porcentajes acumulan son la calidad y espacios, y acceso a la alimentación, con el 15.3 y 20.2 por ciento respectivamente.

La riqueza natural con que cuenta el municipio está representada por 16,300 hectáreas de bosque donde predominan especies de pino, roble, encino y frutales, principalmente, destaca la presencia del Sitio Ramsar No 1466 "Laguna de Zapotlán", Humedal de importancia internacional y corredor migratorio de aves acuáticas de Norteamérica y Centroamérica. Sus recursos minerales son yacimientos de fierro, cal, mármol, yeso y arcilla. Dentro la superficie territorial de 34,389 hectáreas, de las cuales 11,025 son utilizadas con fines agrícolas, 7,283 se utilizan en la actividad pecuaria, 14,226 son de uso forestal y 250 hectáreas son suelo urbano; el resto 1,650 hectáreas, no se específica. En lo que a propiedad se refiere, una extensión de 20,795 hectáreas es propiedad privada y 11,979 es ejidal, y 1,615 hectáreas es no especificada. Destacan los cultivos de aguacate, berries, maíz, garbanzo, avena, agave y sorgo. Cuenta, además. Se cría ganado bovino de carne y leche, porcino, caprino, abejas nativas y colmenas, aves de carne y postura pesca de tilapia, carpa y charal. Se explota principalmente los bosques de pino, encino y tule de la zona lagunar. Dentro de las actividades ancestrales se mantiene la artesanía del tule, la cual la desarrollan 350 familias y la actividad pesquera, realiza por dos sociedades cooperativas con 65 socios, destaca la captura de tilapia (Oreochromis spp.), carpa (Ciprinius carpio), lobina (Micropterus salmoides) y charal (Menidia grandocule). 


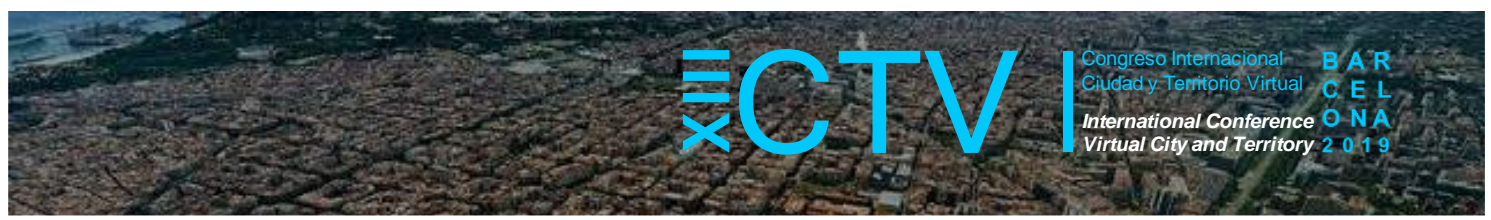

En Tuxpan el 53.3 por ciento de la población se encuentra en situación de pobreza, es decir, 15,550 personas comparten esta situación en el municipio, así mismo el 26.4 por ciento (7,697 personas) de la población es vulnerable por carencias sociales; el 7.4 por ciento es vulnerable por ingresos y 13.0 por ciento es no pobre y no vulnerable. Es importante agregar que el 10.5 por ciento de Tuxpan presentó pobreza extrema, es decir, 3,074 personas, y un 42.7 por ciento en pobreza moderada (12,476 personas). De los indicadores de carencias sociales, destaca que el acceso a la seguridad social es la más alta con un 63.4 por ciento, que en términos absolutos se trata de 18,504 habitantes. Los que menos porcentajes acumulan son calidad y espacios de la vivienda y acceso a los servicios básicos en la vivienda, con el 16.8 y 19.2 por ciento respectivamente.

La riqueza natural con que cuenta el municipio está representada por 7,900 hectáreas de bosque, donde predominan especies de pino, encino, madroño, nogal, pinabete y oyamel. Parte del parque Nacional Nevado de Colima (ANP Federal) corresponde a este municipio y parte del bosque mesofiló (ANP, Estatal). La mayor parte del suelo tiene un uso agrícola y la tenencia de la tierra es predominantemente ejidal. De los cultivos locales destacan: pastos, caña de azúcar, maíz, agave, jícama, chile, sorgo, elote y café, recientemente berries y aguacate. Se cría ganado bovino de carne y leche, porcino, caprino, abejas, aves de carne y postura. En la industria destaca por la Papelera Atenquique que tiene importancia a nivel nacional, existen además las empresas Forestal Alpeña, Los Trapiches, PROENSI, las minas de mármol y pequeñas empresas dedicadas a la costura textil, ladrilleras, paileras, carpinterías.

De los cinco municipios de la zona de estudio, Tuxpan es el municipio que tiene grupos indígenas registrados ante el Instituto Nacional Indigenista (INI) con una lengua indígena Náhuatl existen 362 personas con incrementos graduales de un 8.0 por ciento.

En el municipio de Tuxpan destacan las amenazas generadas por la contaminación del Río Coahuayana, debido a los cambios de uso de suelo que se han hecho en esa cuenca por el alto incremento de cultivo de aguacates, berries y caña de azúcar. Lo cual impacta, el ingenio azucarero no desarrollando buenas prácticas en la cosecha de la caña y su industrialización azucarera, lo cual vierte cantidades significativas de aguas no tratadas, sumándose, el municipio, quien no cuenta con plantas de tratamiento para aguas urbanas, las cuales repercute en el Río Coahuayana impactando su calidad de agua, biodiversidad y actividades productivas.

Los beneficios ambientales, sociales y económicos fueron los siguientes:

- Ambientales: Se contribuye a la protección y funcionalidad de las tres cuencas hidrólogicas, para la generación de recursos hídricos: ciclo del agua, captación, almacenamiento, filtración, purificación y abastecimiento de agua potable a las comunidades del área; regulación climática; captura del $\mathrm{CO}^{2}$; captura de contaminantes; ciclo de los nutrientes; biodiversidad ya que se mantiene el hábitat generando especies faunísticas y florísticas, alimentos básicos en cadena trófica a partir de vegetales y animales comestibles; contribución a la fijación de carbono con la protección y conservación de los ecosistemas; generación de belleza escénica para el trabajo, recreación, descanso y ocio; mitigación de meteoros, sequias y tormentas con avalanchas destructivas.

- Sociales: Cambios de paradigmas educativos, espirituales y religiosos, mejora en la educación, cultura y calidad de vida, sentido de pertenencia e identidad local y regional, fortalecimiento a las redes sociales; contribuir a la adquisición de educación y cultura ambiental para para la protección, conservación y manejo sustentable de los ecosistemas; mejoramiento en las prácticas educativas mediante la educación ambiental para la sustentabilidad, mejoramiento y prevención en el fenómeno salud - enfermedad; cohesión social, respeto mutuo, trabajo en equipo, adquisición de los valores de la sustentabilidad. 


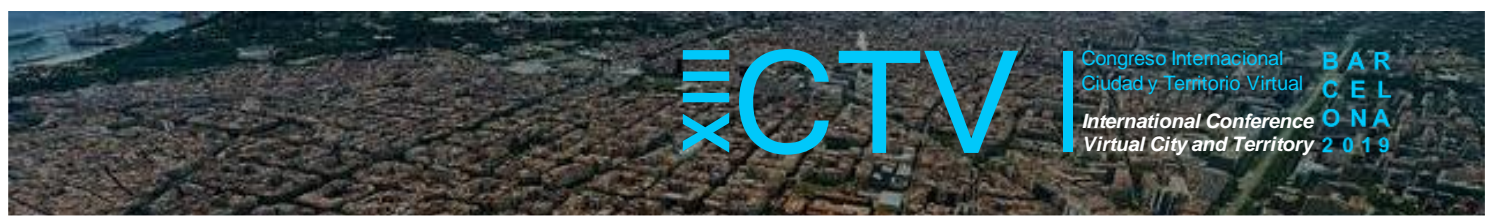

- Económicos: Mejora en las buenas prácticas silvícolas, agrícolas, pecuarias, forestales y turísticas, capacitación en materia gestión ambiental desde lo local a lo global; contribuir a la diversificación de fuentes de empleo con igualdad de género; visión y misión en el buen aprovechamiento económico de los ecosistemas, trasferencia adopción y validación de ecotécnias de bajo impacto ambiental que integren a la familia.

Los participantes adquirieron capacidades adaptativas al cambio climático, para reducir las amenazas naturales, económicas, tecnológicas y productivas mediante la Educación Ambiental formal e informal, lo cual le da los conocimientos útiles, las habilidades, destrezas y los valores necesarios para lograr reducir la vulnerabilidad y contribuir a la adaptación climática para alcanzar la sustentabilidad en su región.

Pasaran del paradigma de involucrados a comprometidos respecto a la adaptación y mitigación del cambio climático con la aplicación de buenas prácticas en el uso y aprovechamiento de los ecosistemas.

Los participantes adquirieron el compromiso de socializar, concientizar y educar a sus familiares, amigos y sociedad donde cohabitan, para propiciar gestiones de políticas públicas y gobernanza que mejore la calidad de vida de su comunidad.

Los participantes deben de comprometerse a sostener los objetivos y las metas alcanzadas para la contribución al cumplimiento del Centro de Educación y Capacitación para el Desarrollo Sustentable (CECADESU) con la formación de una sociedad corresponsable y participativa con educación y cultura de sustentabilidad ambiental ya que es parte fundamental del Plan Nacional de Desarrollo.

\section{Conclusiones}

El proyecto se realizó en tres cuencas hidrológicas del sur de Jalisco, en los municipios de Zapotitlán de Vadillo, Tuxcacuesco, Tuxpan, Zapotlán el Grande y Gómez Farías. La situación de cambio climático y deterioro ambiental está afectando significativamente a los ecosistemas prioritarios que se localizan dentro del área de estudio, por lo cual es una prioridad la adaptación, y mitigación al cambio climático para reducir la vulnerabilidad a que están expuestos la biodiversidad de dichos municipios y esto se puede lograr mediante un proceso educativo y cultural.

En cuanto a vulnerabilidad existe una alta inseguridad social derivada de la delincuencia generada por la corrupción y perdidas de valores y la falta de aplicación de las leyes, normas y reglamentos, la cual la está ocasionando la pobreza, la desigualdad, los bajos niveles de educación, la pérdida de la producción y valores en la sustentabilidad, así como la degradación ambiental. A ello se le suman los eventos naturales tectónicos del sistema volcánico El Colima y el volcán de fuego con alta actividad reciente.

Existe cierto grado de viabilidad que puede contribuir a la resiliencia de la región para contribuir a la remediación del cambio climático, ya que es un problema global en cual se puede contribuir con acciones locales de buenas prácticas en el uso ya aprovechamiento racional de los bienes y servicios ecosistémicos de la región; mediante el desarrollo de capacidades adaptabilidad, mediante la educación ambiental formal e informal, lo cual fortalece y fomenta los valores de la sustentabilidad. 


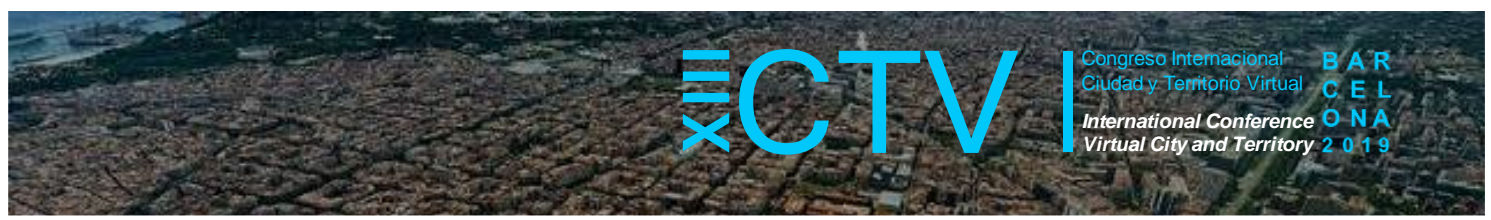

Podemos asumir que quienes se benefician son directamente los grupos de jóvenes estudiantes y comunidad indígena que participan en este proyecto, asimismo, las comunidades enmarcadas en los cinco municipios de la zona de estudio mediante la comunicación entre sus familias, amistades y entorno social.

Ya que mediante estas acciones del programa de educación ambiental se promueve el cambio de paradigmas orientados a la sustentabilidad, el uso racional de los recursos naturales mediante buenas prácticas y el uso de ecotécnias de bajo impacto ambiental acordes a los sistemas productivos.

En el presente trabajo participaron 1,978 personas los cuales son estudiantes de educación básica y de educación media superior, así como productores agropecuarios, forestales y turísticos de los cinco municipios antes mencionados y 50 miembros de la comunidad indígena nahua: estudiantes de la telesecundaria del ejido de Zenzontla, del Municipio de Tuxcacuesco, Escuela preparatoria de Tuxcacuesco, Zapotitlán de Vadillo, Ciudad Guzmán, Tuxpan y escuela secundaria de San Sebastián del Sur, Colegio de bachilleres del Estado de Jalisco (COBAEJ), Centro de Bachillerato Tecnológico Industrial y de Servicios (CBTis).

En los cinco municipios se trabajó con grupos de jóvenes estudiantes de edad entre 13 a 18 años pertenecientes a las instancias educativas gubernamentales de nivel básico y medio superior. Además, con productores agropecuarios, forestales y turísticos, una comunidad indígena presente en el municipio de Tuxpan contando con la participación de mujeres, jóvenes adolescentes y niños.

Esto con la finalidad de cambiar los paradigmas a temprana edad hacia la sustentabilidad de los recursos naturales mediante la implementación de los programas de protección, conservación, manejo y aprovechamiento de los ecosistemas y sus recursos, desarrollando ecotécnias de bajo impacto ambiental, manteniendo una interacción respetuosa entre medio ambiente, sociedad y economía del entorno.

Contribuciones de los autores: J. Guadalupe Michel Parra, ha contribuido en el beneficio social entre humedales naturales y poblaciones vulnerables en Zenzontla, Jalisco. Oziel Dante Montañez Valdez, colabora en la educación ambiental no formal en primarias para preservar el sitio Ramsar 1466, Jalisco, México. José Alejandro Martínez Ibarra, estudia parte de la vigilancia de la presencia de triatómicos mediante gallineros en el sur de Jalisco, México. Tomás Eduardo Orendain Verduzco, apoya en el Programa de Rutas Creativas Estrategias para el Desarrollo Regional que integra el proyecto de Vías Verdes en los municipios. Michel Hernández, Andrés Emmanuel, colabora en los rasgos y aspectos de interés para la conservación en la Laguna de Zapotlán. Cerón Bracamontes, Miriam, asiste en el programa de conservación de los humedales.

Conflicto de Intereses: Los autores declaran que no hay conflicto de intereses.

\section{Bibliografía}

Comisión Nacional del Agua. (2017). Ley de Aguas Nacionales y su reglamento. Artículo 27 Constitucional. México. 


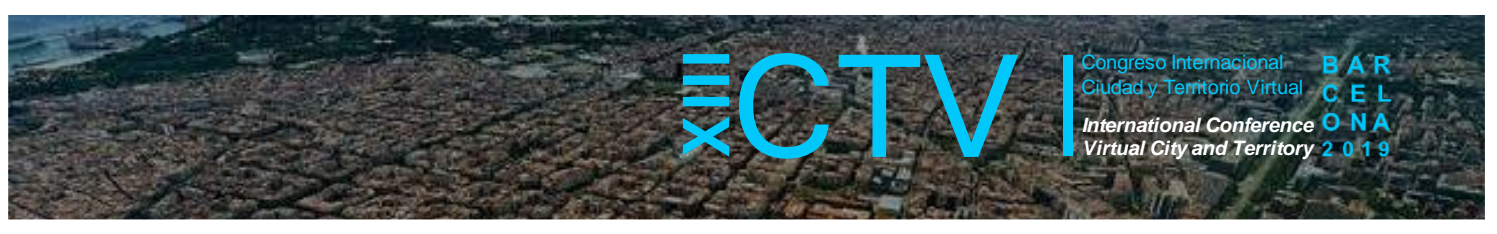

Consejo Nacional de Evaluación de la Política de Desarrollo Social. (2018). Medición de la pobreza. Recuperado de: https://www.coneval.org.mx/Medicion/MP/Paginas/Pobreza-2018.aspx

Constitución Política de los Estados Unidos Mexicanos. Artículo 27 Constitucional (2017) Diario Oficial de la Federación. México.

Ley General del Equilibrio Ecológico y Protección al Ambiente. (2016). Titulo Primero, Art.3, 13, 15, 17. México. D.F.

Michel P J. G. et al.; (2018). Guía para la Protección, Conservación y Manejo de los humedales. Ed No 2. Ed Porrúa Print. México. D. F.

SEMARNAT - SEMADES. (2014) Programa de Ordenamiento Ecológico del Municipio de Zapotlán el Grande. Ciudad Guzmán, Jalisco. 